\title{
CAUDOSPORA SIMULII, N. G., N. SP., MICROSPORIDIE \\ PARASITE DES LARVES DE SIMULIUM
}

\section{Par J. WEISER}

Nous connaissons déjà plusieurs parasites de l'ordre Microsporidies, qui vivent dans les corps adipeux des larves de Simulium. Ce sont des Nosematides comme Thelohania fibrata, T. bracteata, Nosema simulii, Plistophora simulii et $P$. multispora ou des Mrazekides comme Octosporea simulii. Des infections des larves sont très fréquentes, mais on ne trouve, dans la plupart des larves infectées, que des genres déjà connus. Au mois de mai 1944, j'ai trouvé, dans du matériel nouveau, quelques larves de Simulium qui avaient une autre couleur que les larves infectées par des Microsporidies déjà connues. Il $\mathrm{y}$ avait, dans le premier matériel, six larves et, une semaine plus tard, deux nouvelles larves. Pendant tout l'été, on ne rencontra aucune larve présentant cette infection particulière, bien qu'on trouvât beaucoup de larves infectées par d'autres Microsporidies. Jusqu'au mois d'avril de l'année suivante, on n'a pas vu de nouvelles larves avec l'infection particulière. En tout, on a trouvé des larves infectées dans des échantillons de trois localités : dans des ruisseaux près de la ferme Nemojov, près du village Nejevin et dans des canaux de drainage près d'Obolce, toutes localités aux environs de Chotěbor, C.S.R. L'infection est évidemment liée au printemps, peut-être à l'apparition d'une espèce spéciale de larves-hôtes.

Les larves atteintes par cette infection nouvelle se distinguent des larves ayant une autre infection par leur abdomen volumineux et brunâtre. Les parasites remplissent l'abdomen des hôtes et sont entourés par les membranes des corps adipeux comme par une paroi kystique. Le tissu adipeux est le siège propre de l'infection. Les kystes grossissent, remplissent le corps adipeux qu'ils dissolvent, en dilatant les membranes du corps et en produisant des tumeurs sous la cuticule de l'abdomen. Les septa segmentaires des larves s'enfoncent dans les kystes, apparaissant, à travers la chitine de leur cuticule, comme des taches rougeâtres. Les spores comprimées remplissent les kystes, souvent avec le reste du corps adipeux, dans lequel les spores pénètrent de tous les côtés. Les

ANin. de Parasitologie, T. XXII, nos 1-2, 1947, p. 11-15. 
stades trouvés appartiennent à la schizogonie et à la sporogonie du parasite.

Schizogonie. - Comme ehez la plupart des autres Microsporidies (nous allons voir que ce nouvel organisme appartient aux Microsporidies), nous ne trouvons pas le premier stade de la schizogonie, le planonte, non par ce qu'il est très rare, mais parce que les stadès du planonte sont très semblables aux autres stades mononucléés de la schizogonie. Les stades les plus jeunes sont des formes rondes, mononucléées, de $7 \mu$ de diamètre, montrant un noyau clair avec peu de chromatine, sous l'aspect d'un ou plusieurs grains chromatiques (fig. $1, a, d$ ). Ce manque de chromatine caractérise l'organisme pendant toute l'évolution des noyaux. Les schizontes jeunes se divisent par des figures mitotiques analogues aux dicaryons des autres Microsporidies et aux chromosomes évidents de Thelohania opacita et $T$. legeri. En outre, on voit des figures mitotiques fines et difficiles à distinguer. Les chromosomes, au nombre de 14-20, sont de longs rubans, ressemblant aux chromosomes de Plistophora chironomi. La multiplication nucléaire n'étant pas, au début, accompagnée de division cytoplasmique, il se forme des schizontes avec deux noyaux (fig. $1, b, c$ ). Leur chromatine s'amasse dans le centre des noyaux en un grain arrondi et il se forme ainsi des noyaux au repos. Après la division suivante, nous trouvons deux grains chromatiques dans chaque noyau et nous supposons que dans la mitose précédente le noyau s'est divisé en quatre parties : toujours deux noyaux-fils forment deux grains chromatiques dans un noyau au repos, comme un dicaryon d'après Debaisieux (fig. 1, $d, e, f)$. Comme, pendant les mitoses, les deux grains chromatiques se démembrent en filaments de chromosomes qui se mélangent entre eux, nous ne trouvons pas de demi-noyaux des dicaryons aussi dans les mitoses (fig. $1, h$ ).

Sporogonie. - Les stades binucléés ou dicaryons forment, par des multiplications successives (fig. 1, $a-g$ ), des plasmodes avec des noyaux couplés (dicaryons) ou avec des noyaux séparés. Cela

\section{PLANCHE I}

Caudospora simulii. - $a-d$, première division. Zenker-Heidenhain, $\times 2.500$; $e-k$, formation des plasmodes polynucléés, Zenker-Heidenhain, $\times 2.500$; $l, m$, plasmodes colorés par le Giemsa sur frottis desséchés, $\times 2.500 ; n$, dissociation des plasmodes en sporontes ; $o-r$, sporontes colorés par l'hématoxyline ferrique, $\times 2.500 ; s$, un sporonte coloré par le Giemsa, $\times 2.500$. 
ANNALES DE PARASITOLOGIE

T. XXIl, Nos 1-2, 1947
Planche I

(Mémoire J. Weiser)

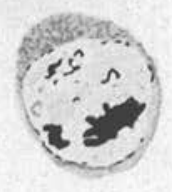

a.
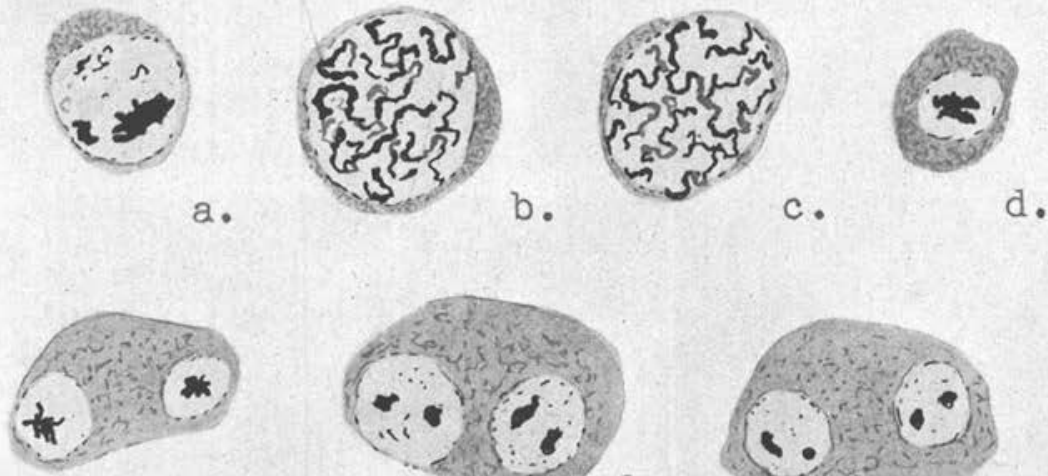

e.
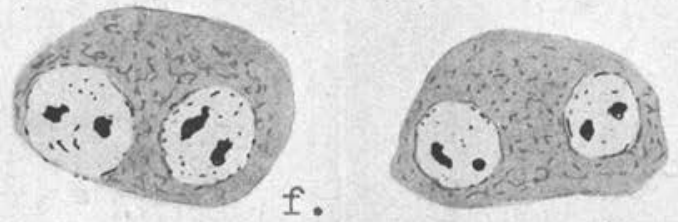

g.

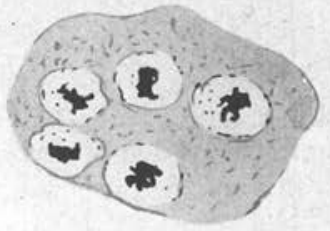

h.
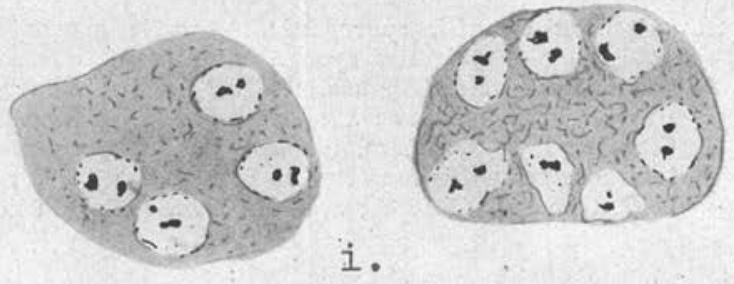

j.
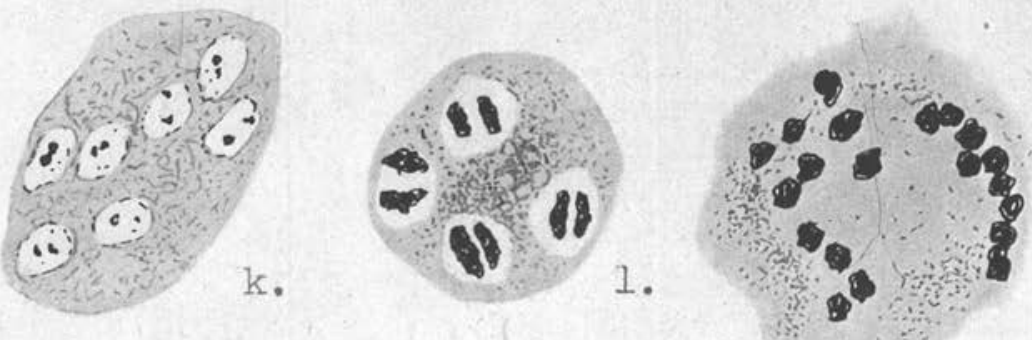

m.
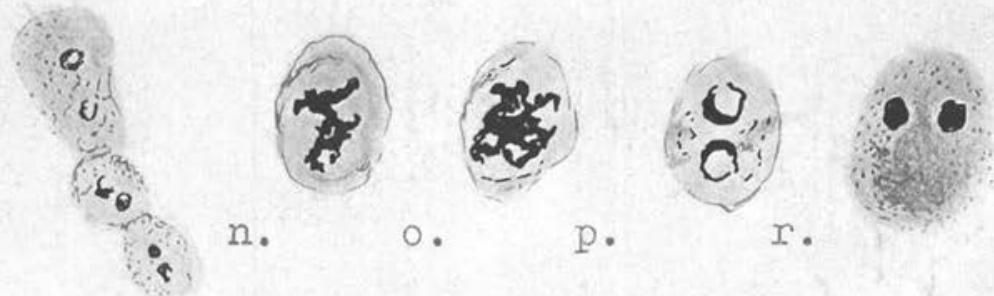

r.

S. 


\section{PLANCHE II}

Caudospora simulii, sporogonie. - $a, b$, sporontes colorés au Giemsa sec, $\times 1.050 ; c, e$, jeunes spores, hématoxyline ferrique, $\times 1.050 ; d, f$, jeunes spores colorées au Giemsa, $\times 1.050 ; g$, jeune sporonte coloré par l'hématoxyline ferrique, $\times 2.500 ; h$, spore mûre in vivo, $\times 2.500 ; c h-k$, filament polaire, Giemsa, $\times 1.005 ; l, m$, réaction de Feulgen ; $n, o, p$, coupe de la spore en trois directions, hématoxyline ferrique, $\times 2.500 ; r, s, t$, spores colorées par le Giemsa sec, $\times 2.500$; $u$, vacuole à la base de la spore. 


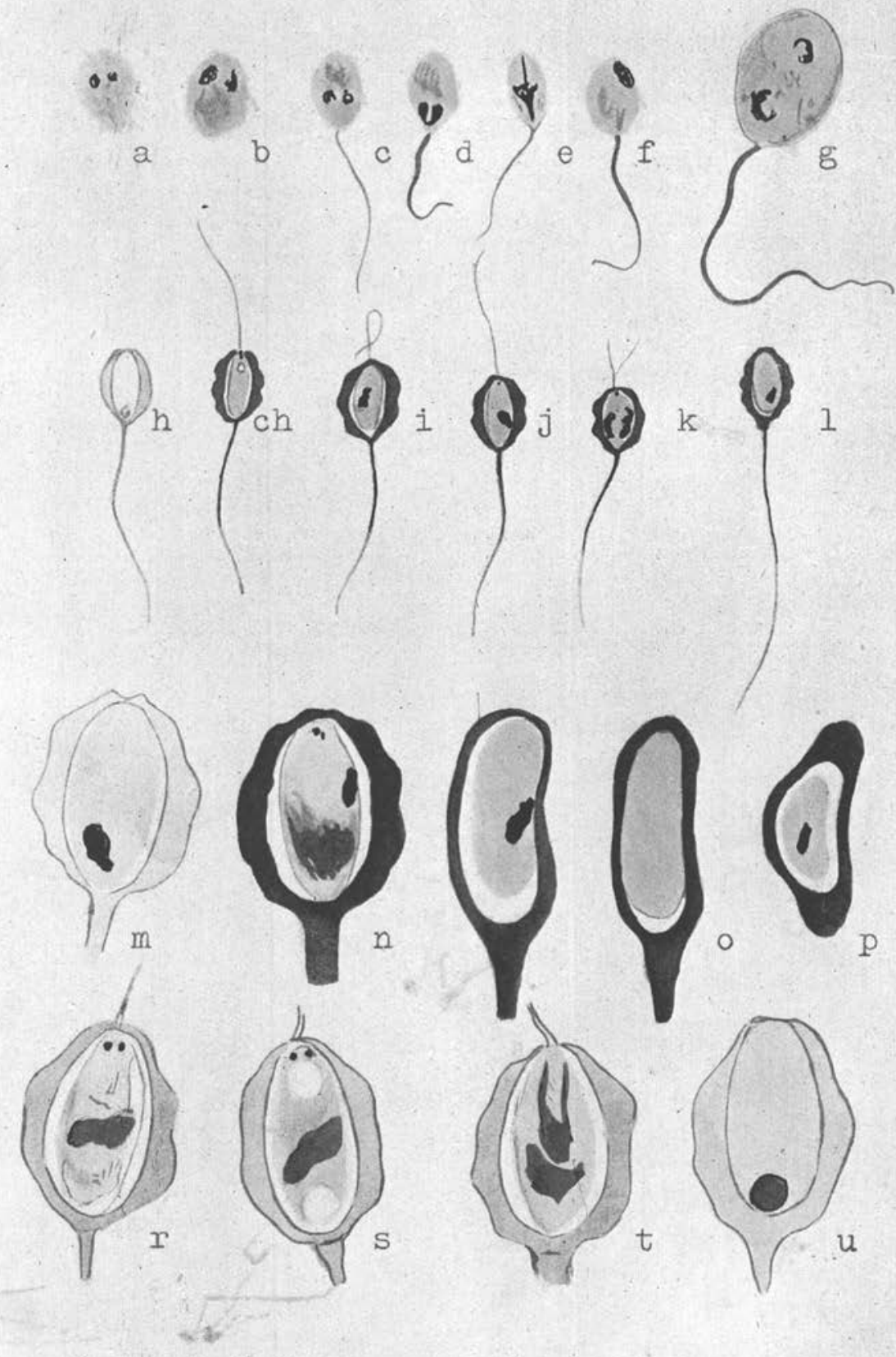


PLANCHE III ( ${ }^{r e}$ partie)

Spores colorées par le Giemsa. - b, vacuoles à la base ; $c$, frottis coloré par le Giemsa humide, après fixation par le Zenker. 
ANNALES DE PARASITOLOGIE

T. XXII, Nos $1-2,1947$
Pianche III (1 ${ }^{\text {re }}$ partie)

(Mémoire J. Weiser)
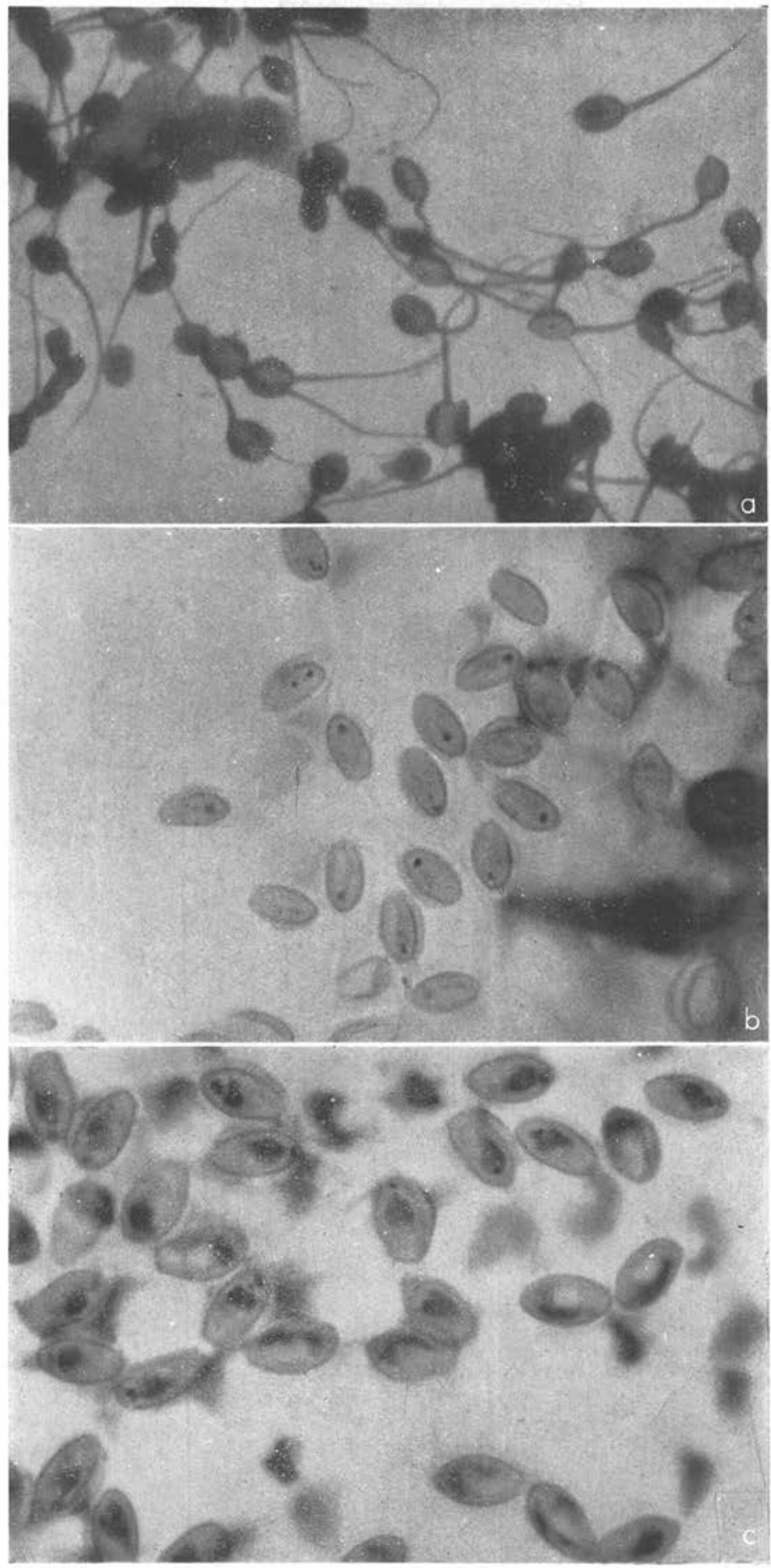

Masson et Cie, Editeurs 
PLANC̣HE III ( $2^{\circ}$ partie)

Spores colorées par le Giemsa. - $d-f$, stades végétatifs et spores mûres dans des coupes de kystes (Zenker, hématoxyline ferrique). 
ANNALES DE PARASITOLOGIE

T. XXII, Nos 1-2, 1947
Planche III (2e partie)

(Mémoire J. WeIser)
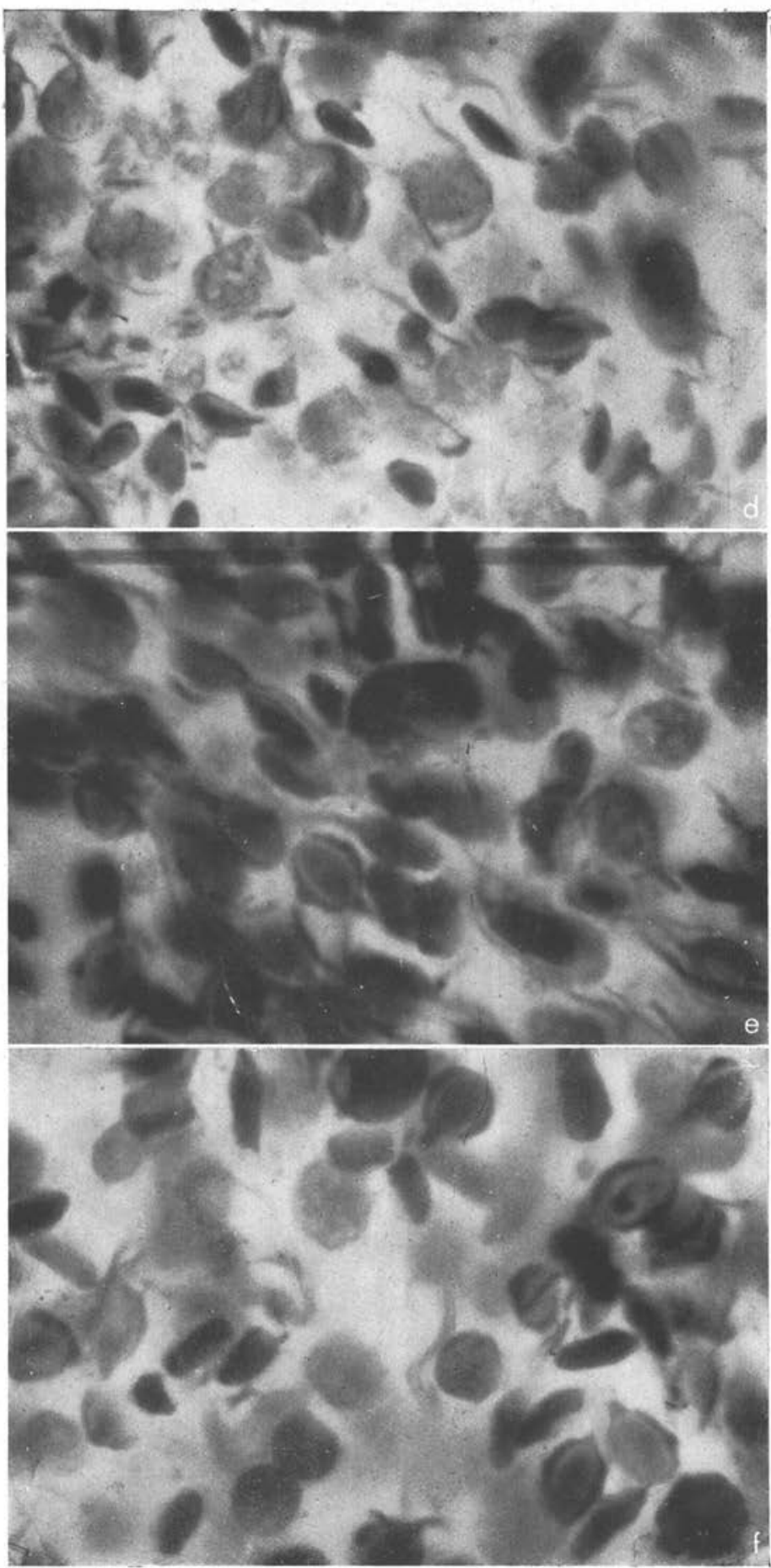

Masson et Cio, Editeurs 

dépend de la méthode de coloration et de fixation. Dans les irottis desséchés, colorés par le Giemsa (fig. $1, l, m$ ), nous ne trouvons que le deuxième type, avec des noyaux solitaires. Un plasmode peut montrer jusqu'à 18,24 ou 30 noyaux, cela correspond à 9,12 ou 15 dicaryons sur les frottis fixés au Zenker et colorés par l'hématoxyline ferrique d'Heidenhain. La moyenne des plasmodes montre seulement 4-7 dicaryons. Puis le plasmode arrondi, mesurant 15-30 $\mu$ de diamètre, se dissocie et chaque élément binucléé, provenant de cette dissociation, deviendra un sporoblaste (fig. $1, n, o, p$, $r, s)$. Chaque sporoblaste donne sur place une spore. Pendant la transformation suivante du sporoblaste, nous trouvons un phenomène intéressant. Sur un pôle de la spore jeune se forme un filament (fig. 2, $c, d, f$ ). La provenance et la formation de ce filament n'ont pu être suivis. Il est long de 7 à $10 \mu$, mou, immobile et courbé en $\mathrm{S}$. Les deux noyaux de la spore sont, à ce stade, situés l'un à côté de l'autre ; dans d'autres cas ils sont joints par une commissure en forme d'haltère (fig. $2, c, d$ ). Dans la suite, le sporoblaste, mesurant $3,5,4,5 \times 3 \mu$, s'entoure d'une membrane rigide, le filament devient plus résistant et droit et il se forme une cauda, à cause de cela, je nomme ce parasite Caudospora. La spore mûre mesure 4,5-5 $\mu$ de longueur sur $5 \mu$ de largeur, l'appendice caudal lui-même mesure $14 \mu$ et dans plusieurs cas jusqu'à 20-24 $\mu$ (fig. $2, h-l$ ). La spore est aplatic dorsoventralement (fig. 2,0 ), avec deux rachis le long des côtes (fig. $2, F$ ). Les rachis sont lisses sur le vivant (fig. 2, $h$ ). Après la fixation, ils se rident et se recourbent (fig. 2, Ch-u). La base de la cauda forme toutefois un petit élargissement duquel sort l'appendice entier (fig. $2, l, o$ ).

Dans l'intérieur de la spore, de forme elliptique, nous trouvons, à l'extrémité postérieure, près de la cauda, une petite vacuole qui se colore par le Giemsa comme un grain métachromatique. Par la réaction de Feulgen nous identifions, près de la base de la spore, un petit granule rouge (fig. $2, m, n$ ), pas très distinct, en corrélation avec la pauvreté en chromatique à tous les stades de l'évolution du parasite. A l'extrémité antérieure de la spore, on colore par le Giemsa un ou deux grainis métachromatiques et plus en arrière une masse correspondant au filament polaire enroulé, le reste de la spore est occupé par le cytoplasme (fig. 2, $n$, $r-t$ ). Ce filament mesure $6,8,12 \mu$ de longueur et il est à peu près quatre fois plus mince que la cauda. Pour l'évagination du filament il suffit d'une légère pression ou d'un changement de la concentration osmotique du milieu pendant le séchage du frottis. Dans un cas unique, nous avons trouvé deux filaments polaires (fig. 2, $k$ ), mais, 
dans ce cas, le noyau aussi était dédoublé et cela devait représenter une anomalie, bien connue chez les Microsporidies sous le nom de macrospore. Nous n'avons jamais trouvé de spores vides permettant de voir le pore, mais la membrane du pôle apical de la spore était très mince et perforée par le filament polaire, aussi je suppose que le pore se trouve à cet endroit (fig. $2, m, r-u$ ).

En suivant l'évolution de ce parasite que je nomme Caudospora simulii, n. sp., nous voyons qu'elle correspond à celle des Microsporidies. La schizogonie, la sporogonie, la première division avec de grands chromosomes, les dicaryons, l'organisation de la spore, y compris le filament polaire, et aussi le siège de l'infection, tout y correspond. Mais nous trouvons aussi quelques caractères différents, comme la spore aplatie, les rachis latéraux, une cauda à l'extrémité postérieure. Tous ces caractères ne se trouvent jamais réunis chez aucun autre Microsporidie. L'évolution de la Caudospora correspond à celle de Nosema ou Plistophora.

Nous trouvons une analogie à la cauda de Caudospora chez quelques Mrazekides. Ici l'appendice caudal se constitue à l'extrémité postérieure des spores jeunes, sous forme d'un prolongement pointu, large et aplati, qui durcit au cours de l'évolution et subsiste comme un petit appendice aplati à l'extrémité de la spore mûre. Chez Caudospora nous trouvons un filament fin, souple et flottant, à l'extrémité de la spore. Chez les Microsporidies il y a, en dehors des Mrázekides (Mrázekia brevicauda, M. caudata, M. lumbriculi, M. ilyodrilli, M. limnodrilli), des différentiations ressemblant à la cauda de Caudospora chez Thelohania octospora. Chez cette espèce, Goodrich (1920) a coloré par le Lugol trois appendices de $20 \mu$ de longueur à chaque spore. Ces appendices, fins et aplatis, sont plus épais que celui de Caudospora. Dans un autre cas, chez Trichoduboscquia epeori, Léger trouvait, sur chaque pansporoblaste, quatre aiguillons implantés dans la membirane de ce pansporoblaste. Dans ce cas aussi, les appendices se colorent difficilement et ils ne présentent avec la membrane des spores aucune connection. Nous trouvons des spores avee un appendice semblable chez d'autres protozoaires, par exemple Barrouxia caudata, chez un Eimeridé de Lithobius martini (spores 8-10 $\mu$ long.) et chez des spores de Myxosporidies du genre Henneguya.

Notre forme est si différente des autres genres des Nosématides 'fue nous la plaçons dans la famille des Nosématidés, à laquelle elle appartient par sa spore ovale ou elliptique, dont la longueur est moins de quatre fois la largeur, comme genre nouveau, Caudospora. En suivant l'énumération de Kudo (1924), pour la famille des Nosé- 
matidés, nous plaçons ce nouveau genre Caudospora comme neuvième genre, après le huitième genre Plistophora.

\section{Diagnose :}

Genre Caudospora: Microsporidie dont chaque pansporoblate donne finalement plusieurs sporontes. Les spores sont ovales et aplaties avec un prolongement caudal.

Espèce type : Caudospora simulii : Les plasmodes peuvent montrer jusqu'à 15 dicaryons. Les sporoblastes mesurent 3,5-4,5 $\times 3 \mu$, avec un filament de 7-10 $\mu$. Les spores mûres, aplaties, mesurent 4,5-5 $\times 5 \mu$, l'appendice caudal 14-24 $\mu$. Deux rachis le long des côtés de la spore. Filament polaire de 6-12 $\mu$ de longueur.

Habitat : Corps adipeux des larves de Simulium sp.

Localités : Nemojov, Nejepin, Obolce dans les environs de Chotěboř, Tchécoslovaquie.

\section{BIBLIOGRAPHIE}

Drbaisieux (P.) et Gastaidi (L.). - Les Microsporidies parasites des larves de Simulium. II. La Cellule, XXX, 1919, p. 325-336.

Goodrich (H. L. P.). - The spore of Thelohania, Arch. zool. exper., LIX, 1920. Jínovec (O.). - Revision der in Simulien-Larven parasitierenden Mikrosporidien, Zool. Anz., CXLII, 1943, p. 173-179.

Kudo (R. R.). - A biologic and taxonomic study of the Microsporidia. Illinois Biological Monographs, 1924.

LÉGer (L.). - Sur Trichoduboscquia epeori Léger, Microsporidie parasite des larves d'Ephémérides. Trav. lab. hydrobiol. Pis. Unin., Grenoble, XIV, 1927. Strickland (E. H.). - Further observations on the parasites of Simulium larvae. Jour. Morphol., XXIV, 1913, p. 43-102.

Werser (J.). - Zur Kenntnis der Mikrosporidien aus Chironomiden-Larven, Zool. Anz., CXL, II, 1942, et CXLI, p. 255-264.

- Př́ispẻvek k znalosti mikrosporidii perlooček a buchanek. Práce Mor. prír. společnosti, XVII, 1945, 1.

- Studie o mikrosporidiích z larev hmyzu našich vod. Věstník čsl. zoolog. společnosti, 10, XIII, 1946.

\section{Laboratoire de Parasitologie de la Faculbé des Sciences de l'Université Charles, Prague, Tchécoslovaquie}

